\title{
Langmuir
}

pubs.acs.org/Langmuir

(C) 2009 American Chemical Society

\section{A Study of the Dynamic Interaction of Surfactants with Graphite and Carbon Nanotubes using Fmoc-Amino Acids as a Model System}

\author{
Yanning Li,${ }^{\dagger}$ Brian G. Cousins,${ }^{\dagger}$ Rein V. Ulijn, ${ }^{*}, *$ and Ian A. Kinloch*, ${ }^{*}$ \\ ${ }^{\dagger}$ School of Materials, University of Manchester, Manchester, U.K., M1 7HS, and ${ }^{\$}$ West CHEM, Department of \\ Pure \& Applied Chemistry, University of Strathclyde, Glasgow, U.K., G1 IXL
}

Received April 2, 2009. Revised Manuscript Received July 13, 2009

\begin{abstract}
We have studied the dynamic interaction of surfactants with carbon surfaces by using a series of Fmoc( $N$-(fluorenyl-9-methoxycarbonyl)) terminated amino acid derivatives (Fmoc-X, where $\mathrm{X}$ is glycine, tyrosine, phenylalanine, tryptophan, or histidine) as a model system. In these systems, highly conjugated fluorenyl groups and aromatic amino acid side chains interact with the carbon surface, while carboxylate groups provide an overall negative charge. Ideal carbon surfaces were selected which possessed either predominantly macroscale (graphite) or nanoscale features (multiwalled carbon nanotube (MWNT) mats). The adsorption equilibrium for the Fmoc-X solutions with the graphitic surfaces was well-described by the Freundlich model. When a library containing various Fmoc-X compounds were exposed to a target graphite surface, Fmoc-tryptophan was found to bind preferentially at the expense of the other components present, leading to a substantial difference in the observed binding behavior compared to individual adsorption experiments. This approach therefore provides a straightforward means to identify good surfactants within a library of many candidates. Finally, the fully reversible nature of Fmoc-X binding was demonstrated by switching the surface chemistry of carbon substrate through sequential exposure to surfactants with increasing binding energies.
\end{abstract}

\section{Introduction}

The interaction of biological molecules with carbonaceous surfaces is important for a range of applications, including sensors, ${ }^{1-5}$ stents $^{6}$ and purification of blood. ${ }^{7}$ In recent years there has also been an increasing focus on using new types of nanoscale carbon particles, including carbon nanotubes (CNTs). Importantly, in these cases, the use of biomolecular derived surfactants also opens new routes to the dispersion and selfassembly of the nanocarbons, including the introduction of switchable chemistries. Two approaches have been reported toward interfacing CNTs with biological molecules: (i) covalent side-wall functionalization, ${ }^{8,9}$ and (ii) noncovalent adsorption of

*Corresponding authors. (I.A.K.) E-mail: ian.kinloch@manchester.ac.uk. Telephone: +44-161-3063615. (R.V.U.) E-mail: rein.ulijn@strath.ac.uk. Telephone: +44-141-5482110.

(1) Gorodetsky, A. A.; Barton, J. K. J. Am. Chem. Soc. 2007, 129, 6074-6075.

(2) Jonsson-Pettersson, G. Eletroanalysis 1991, 3, 741-750.

(3) Yang, H.; Fung, S. Y.; Sun, W.; Mikkelsen, S.; Pritzker, M.; Chen, P. Biotechnol. Prog. 2008, 24, 964-971.

(4) Cui, Y.; Wei, Q.; Park, H.; Lieber, C. M. Science 2001, 293, 1289-1292.

(5) Gooding, J. J.; Wibowo, R.; Liu, J.; Yang, W.; Losic, D.; Orbons, S.; Mearns, F. J.; Shapter, J. G.; Hibbert, D. B. J. Am. Chem. Soc. 2003, 125, 90069007.

(6) Westwood, A.; Arulrajah, A.; Collins, S.; Rand, B.; Anson, T.; Mikhalovsky, S.; Mikhalovska, L.; Santin, M. Carbon in Health, Environment and Energy, Manchester, 2009 .

(7) Howell, C. A.; Sandeman, S. R.; Phillips, G. J.; Lloyd, A. W.; Davies, J. G.; Mikhalovsky, S. V.; Tennison, S. R.; Rawlinson, A. P.; Kozynchenko, O. P.; Owen, H. L. H.; Gaylor, J. D. S.; Rouse, J. J.; Courtney, J. M. Biomaterials 2006, 27, 5286-5291.

(8) Chattopadhyay, D.; Galeska, I.; Papadimitrakopoulos, F. J. Am. Chem. Soc. 2003, 125, 3370-3375.

(9) Boul, P. J.; Liu, J.; Mickelson, E. T.; Huffman, C. B.; Ericson, L. M.; Chiang, I. W.; Smith, K. A.; Colbert, D. T.; Hauge, R. H.; Magrave, J. L.; Smalley, R. E. Chem. Phys. Lett. 1999, 310, 367-372.

(10) Bandyopadhyaya, R.; Nativ-Roth, E.; Regev, O.; Yerushalmi-Rozen, R. Nano Lett. 2002, 2, 25-28.

(11) Tsang, S. C.; Davis, J. J.; Green, M. L. H.; Hill, H. A. O.; Leung, Y. C.; Sadler, P. J. J. Chem. Soc., Chem. Commun. 1995, 1803-1804.

(12) Davis, J. J.; Green, M. L. H.; Hill, H. A. O.; Leung, Y. C.; Sadler, P. J.; Sloan, J.; Xavier, A. V.; Tsang, S. C. Inorg. Chim. Acta 1997, 272, 261-266. biomolecules including carbohydrates, ${ }^{10}$ proteins, ${ }^{11,12}$ and nucleic acids. ${ }^{13}$ The modification of nanotubes by these biomolecules, as well as their analogs and precursors (such as oligosaccharides, amino acids, peptides, etc.) represents a significant step toward the application of CNTs in the field of biotechnology and the transfer of biomolecular self-assembly techniques to nanomaterials. $^{14}$

Compared with covalent functionalization, noncovalent binding, which is based on $\pi-\pi$ stacking, ${ }^{15-17}$ hydrophobic interactions $^{18,19}$ and electrostatic adsorption, ${ }^{20}$ has less effect on the structural and functional properties of both the nanotubes and the biomolecules. Synthetic aromatic ligands such as pyrene and its derivatives were reported to be able to assemble onto CNTs' surfaces via $\pi-\pi$ stacking interaction. ${ }^{21-26}$ Recently biological molecules containing aromatic residues were also used for CNT

(13) Zheng, M.; Jagota, A.; Semke, E. D.; Diner, B. A.; McLean, R. S.; Lustig, S. R.; Richardson, R. E.; Tassi, N. G. Nat. Mater. 2003, 2, 338-342.

(14) Gazit, E. Chem. Soc. Rev. 2007, 36, 1263-1269.

(15) Chen, R. J.; Zhang, Y.; Wang, D.; Dai, H. J. Am. Chem. Soc. 2001, 123, 3838-3839.

(16) Pender, M. J.; Sowards, L. A.; Hartgerink, J. D.; Stone, M. O.; Naik, R. R. Nano Lett. 2006, 6, 40-44.

(17) Zorbas, V.; Smith, A. L.; Xie, H.; Ortiz-Acevedo, A.; Dalton, A. B.; Dieckmann, G. R.; Draper, R. K.; Baughman, R. H.; Musselman, I. H. J. Am. Chem. Soc. 2005, 127, 12323-12328.

(18) Kong, J.; Franklin, N. R.; Zhou, C.; Chapline, M. G.; Peng, S.; Cho, K.; Dai, H. Science 2000, 287, 622-625.

(19) Valenti, L. E.; Fiorito, P. A.; García, C. D.; Giacomelli, C. E. J. Colloid Interface Sci. 2007, 307, 349-356.

(20) Liu, S.; Cai, C. J. Elem. Chem. 2007, 602, 103-114.

(21) Nakashima, N.; Tomonari, Y.; Murakamiy, H. Chem. Lett. 2002, 638.

(22) Petrov, P.; Stassin, F.; Pagnoulle, C.; Jérome, R. Chem. Commun. 2003, 2904-2905.

(23) Fifield, L. S.; Dalton, L. R.; Addleman, R. S.; Galhotra, R. A.; Engelhard,

M. H.; Fryxell, G. E.; Aardahl, C. L. J. Phys. Chem. B 2004, 108, 8737-8741.

(24) Artyukhin, A. B.; Bakajin, O.; Stroeve, P.; Noy, A. Langmuir 2004, 20 , 1442-1448.

(25) Tomonari, Y.; Murakami, H.; Nakashima, N. Chem.-Eur. J. 2006, 12, 4027-4034.

(26) Nakashima, N. STAM 2006, 7, 609-616. 
functionalization. ${ }^{15-17}$ Wang et al. ${ }^{27}$ employed the phage display technique to search for the peptides with selective affinity for CNTs. They found that the binding sequences were invariably rich in histidine (His) and tryptophan (Trp), with Trp in particular, interacting strongly with the nanotubes' surfaces. As alternative to these expensive, long peptides, we have previously utilized $N$-(fluorenyl-9-methoxycarbonyl) (Fmoc-) terminated aromatic amino acids, which are a combination of synthetic ligands and biomolecules, ${ }^{28}$ to prepare CNTs dispersions. ${ }^{29}$

The majority of the research described above on functionalizing carbon nanotubes through noncovalent approaches has tended to focus on the behavior of the nanotubes, and have not studied the biomolecule's adsorption process. For example, when noncovalent surfactants are used the concentration of the nanotubes that are dispersed is measured but the adsorption kinetics of the surfactant on the nanotubes is not considered. The kinetics and equilibrium of the surfactant are important, though, as if the interaction energy is low then the surfactant may desorb when placed in excess solvent causing the dispersion to break down. Also, the kinetics of the surfactant adsorption may be impractically slow. For example Cathcart et al. suggested that DNA needed to be fully dehybridized prior to fully coating a nanotube, which took up to 50 days under their experimental conditions. ${ }^{30}$ The importance of the adsorption process of the surfactants on the nanotubes is also applicable to more conventional surfactants. For example, in the case of absorbed poly aromatic acid surfactants, the introduction of a base converts the surfactants into their conjugate salts, improving the surfactants' solubility in water and leading to the desorption of the surfactant from the nanotubes. ${ }^{31,32}$ Motivated by this issue of the behavior of the noncovalent functional molecules and surfactants we have investigated the dynamic adsorption of bimolecular derived surfactants on carbonaceous surfaces. Fmoc terminated amino acids (Fmoc-X) were used a model system in which to develop a several of techniques in which the adsorption process could be studied. These techniques can then be applied to other surfactant and noncovalent systems. Mats of aligned carbon nanotubes and graphite were selected as model surfaces as they were immobilized and hence allowed the adsorption process of the surfactants to be studied easily. Furthermore the nanotubes had predominantly nanoscale features, where the graphite had predominantly macroscale features. The aims of this model system were to (1) measure the adsorption kinetics and equilibrium of a range of different Fmoc-Xs and fit their behavior to an isothermal model, (2) develop and assess the use of a library of Fmoc-Xs to evaluate the binding capacity of each component efficiently through competitive binding, and (3) establish if the interaction of the Fmoc-X with the carbon surfaces was really dynamic through the sequential functionalization of a graphite surface.

\section{Experimental Section}

Adsorption Study of Fmoc-X on Graphitic Surfaces. Graphite (EC4, Graphite Technologies Plc.) and MWNT mats

(27) Wang, S.; Humphreys, E. S.; Chung, S. Y.; Delduco, D. F.; Lustig, S. R.; Wang, H.; Parker, K. N.; Rizzo, N. W.; Subramoney, S.; Chiang, Y. M.; Jagota, A. Nat. Mater. 2003, 2, 196-200.

(28) Zhang, Y.; Gu, H.; Yang, Z.; Xu, B. J. Am. Chem. Soc. 2003, 125, 13680 13681

(29) Cousins, B. G.; Das, A. K.; Sharma, R.; Li, Y.; McNamara, J. P.; Hillier, I. H.; Kinloch, I. A.; Ulijn, R. V. Small 2009, 5, 587-590.

(30) Cathcart, H.; Nicolosi, V.; Hughes, J. M.; Blau, W. J.; Kelly, J. M.; Quinn, S. J.; Coleman, J. N. J. Am. Chem. Soc. 2008, 130, 12734-12744.

(31) Verdejo, R.; Lamoriniere, S.; Cottam, B.; Bismarck, A.; Shaffer, M. Chem. Commun. 2007, 513-515.

(32) Wang, Z.; Shirley, M. D.; Meikle, S. T.; Whitby, R. L. D.; Mikhalovsky, S. V. Carbon 2009, 47, 73-79. were selected as model surfaces with macroscale and nanoscale features respectively. The MWNT mats were manufactured inhouse by an injection chemical vapor deposition (CVD) method using previous published protocols. ${ }^{33,34}$ The CNTs were grown for $60 \mathrm{~min}$ at $760{ }^{\circ} \mathrm{C}$ by injecting a solution of $5 \mathrm{wt} \%$ ferrocene in toluene into an argon carrier gas flowing at $100 \mathrm{~mL} / \mathrm{min}$ and preheated to $200^{\circ} \mathrm{C}$. The carrier gas then swept the reactants into the hot zone of the furnace, where the nanotubes grew from silicon oxide growth substrates. The surface area of both the graphite substrates and the MWNT mats was measured using the BET method. Four Fmoc-protected aromatic amino acids (Fmoc-X) were studied; whereby X corresponds to tryptophan (Trp), phenylalanine (Phe), tyrosine (Tyr), or histidine (His). Glycine (Gly) was also used as a nonaromatic control (Figure 1).

The adsorption of the Fmoc-X from solution was monitored using UV-spectroscopy (Figure 1a). The graphite was cut into square substrates $((10 \pm 0.5) \times(10 \pm 0.5) \times(2 \pm 0.1) \mathrm{mm})$ with a mass of $1.8 \pm 0.1 \mathrm{~g}$. These graphite substrates were placed into a semimicro cuvette containing $1 \mathrm{~mL}$ of $0.03 \mathrm{mM}$ Fmoc-X solution using a PBS buffer $(\mathrm{pH}=7.4$, the concentration of PBS buffer was $10 \mathrm{mM}$ ). UV-vis measurements were then performed over time to monitor the adsorption process (Hitachi U-1800 UV-vis spectrometer). The spectra were also collected in the absence of graphite as a control.

The aligned MWNTs were found to absorb more material per unit weight than the graphite, so a higher concentration of Fmoc-X solution had to be used. The MWNT mats were grown on both sides of silicon wafer $((5 \pm 0.1) \times(5 \pm 0.1) \mathrm{mm})$ (Figure 1b) and placed into a cuvette containing $3 \mathrm{~mL}$ of $0.05 \mathrm{mM}$ Fmoc-X solution using a PBS buffer. UV-vis measurements were performed over time with and without the nanotubes being present.

The Freundlich Adsorption Isotherm for Fmoc-X on Graphite. To obtain the adsorption isotherm for Fmoc-X on graphite, a series of Fmoc-Trp-OH solutions with concentrations of $0.02,0.03,0.04,0.06$, and $0.08 \mathrm{mM}$ were prepared. Graphite samples were then placed into $1 \mathrm{~mL}$ of each of the solutions and UV-vis measurements were carried out over time until no further change in the concentration was observed. The same measurements were also conducted with Fmoc-Gly-OH.

Study of the Competitive Binding between Fmoc-Xs on Graphite. Competitive binding experiments were developed to evaluate the strongest binder from a library of Fmoc-Xs. The experiments relied on the ability of the stronger binding Fmoc-Xs to displace other Fmoc-Xs which bound more weakly from the graphite. Therefore over time, the strongest binding Fmoc-X would be predominantly on the surface of the graphite whereas the weaker binding Fmoc-Xs would be predominantly in solution.

The library used consisted of 5 different Fmoc-Xs $(\mathrm{X}=$ Trp $-\mathrm{OH}, \mathrm{Phe}-\mathrm{OH}, \mathrm{Tyr}-\mathrm{OH}$, His $-\mathrm{OH}$ and $\mathrm{Gly}-\mathrm{OH}$ ) all at a concentration of $0.08 \mathrm{mM}$ in PBS. Six graphite substrates were then placed into $150 \mathrm{~mL}$ of the library and the concentration of each individual Fmoc-X species in solution was measured over time. These individual concentrations were measured by taking an aliquot, separating the Fmoc-X species through reverse-phase HPLC and then measuring the absorption of each species at $265 \mathrm{~nm}$ (UltiMate 3000 Intelligent LC system with Acclaim 120 silica-based reversed-phase columns and UVD 170U detector, DIONEX). The Beer-Lambert absorption coefficient of the Fmoc-Xs varied with molecule structure so the initial library with known concentrations was used as a reference for dynamic studies.

Alteration of the Hydrophobicity of HOPG Surface and the Sequential Functionalization with Fmoc-X. Contact angle measurements were conducted to determine the change in the hydrophobicity of HOPG surface upon functionalized with

(33) Singh, C.; Shaffer, M. S. P.; Windle, A. H. Carbon 2003, 41, 359-368.

(34) Singh, C.; Shaffer, M.; Kinloch, I.; Windle, A. H. Phys. B: Condens. Matter 2002, 323, 339-340. 


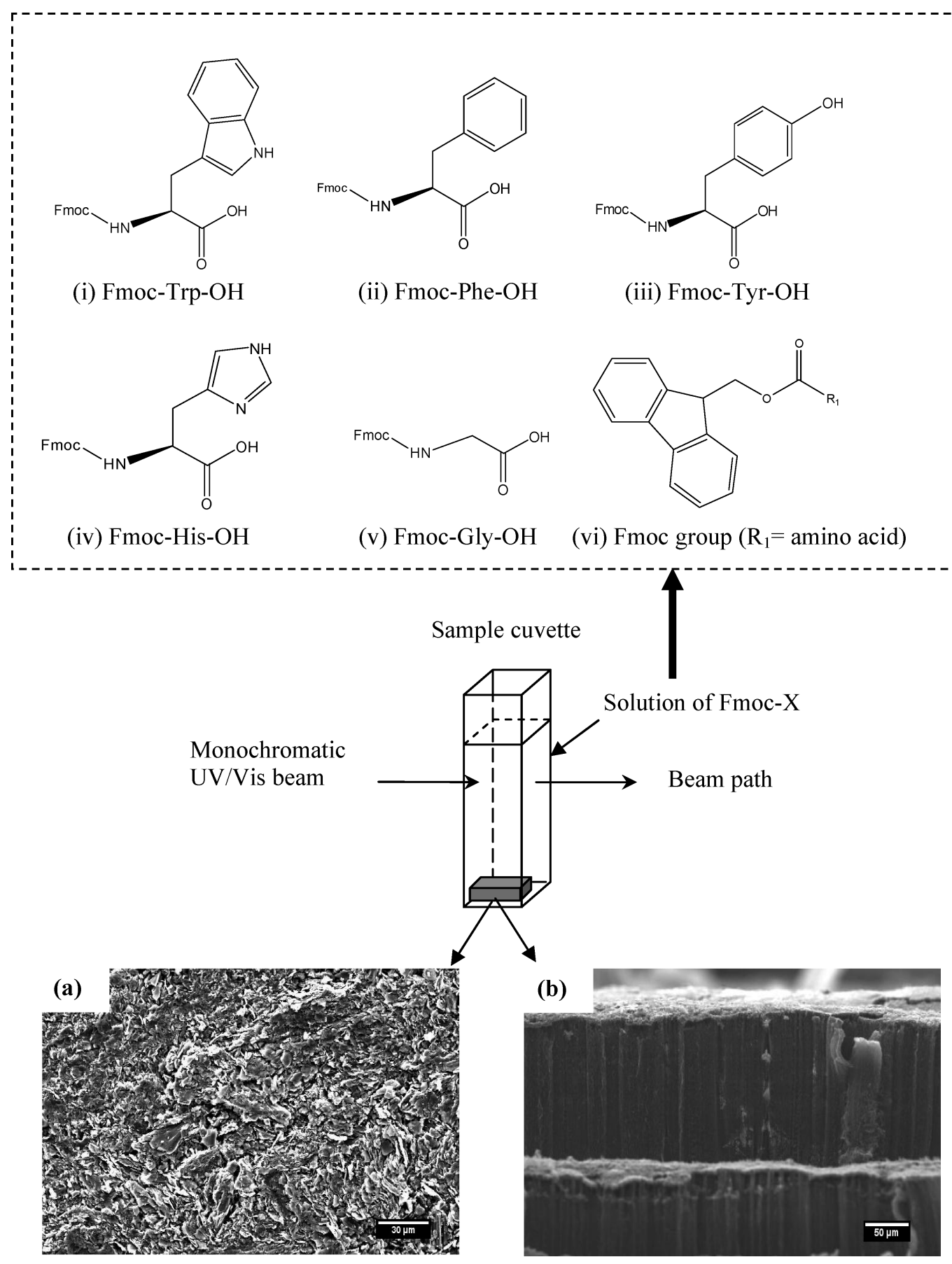

Figure 1. Scheme illustrating the UV-vis measurement of the adsorption of the five Fmoc-Xs solutions on (a) graphite (top view SEM image) and (b) MWNT mats (side-view SEM image, highly aligned MWNT arrays).

Fmoc- Gly-OH and Fmoc-Trp-OH (Krüss GmbH). A freshly cleaved HOPG surface which was approximately atomically flat was obtained by peeling off the topmost layers using scotch tape. A sessile drop of water $(10 \mu \mathrm{L})$ was deposited on the control and the functionalized HOPG surfaces and the images of the water drop were then taken. The measurements were repeated at three different locations on each of the surfaces.

The result obtained above served as a reference to study the displacement of previously adsorbed Fmoc-Gly-OH by Fmoc-Trp-OH on the surface. First the HOPG control sample was placed in $3 \mathrm{~mL}$ of $0.04 \mathrm{mM}$ Fmoc-Gly-OH solution for 2 days and allowed to dry at ambient temperature for contact angle measurement. It was then transferred to $3 \mathrm{~mL}$ of $0.04 \mathrm{mM}$ Fmoc-Trp-OH solution for another 2 days after which the same measurement was carried out.

\section{Results and Discussion}

The Adsorption of Fmoc-X on Graphite Surface. The $\mathrm{UV}$-vis spectra of a series of Fmoc-X solutions were recorded over time both in the absence and presence of graphite. The absorbance at $264 \mathrm{~nm}$, corresponding to the absorbance of Fmoc group, was used to follow the changes in the concentration of the Fmoc-X solutions. As shown in Figure 2a the concentration of Fmoc-Trp-OH control solution remained constant over time suggesting that precipitation and settling of the Fmoc-Trp-OH did not occur. The concentration of Fmoc-Trp-OH solution in the presence of the graphite declined significantly during the first $200 \mathrm{~min}$, after which the rate of change slowed and eventually reached equilibrium. Similar profiles were obtained for the other 

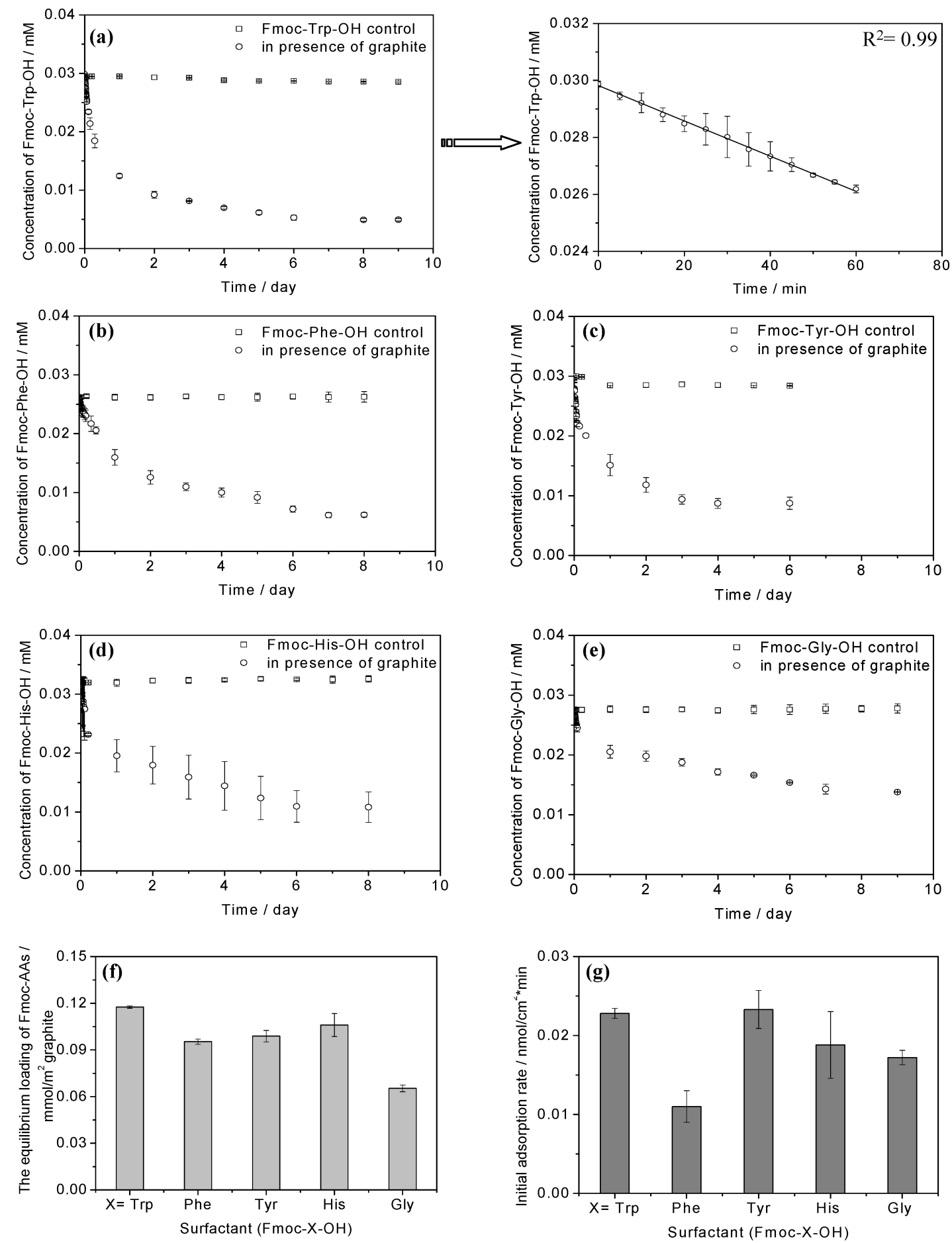

Figure 2. Adsorption profiles of the Fmoc-Xs on graphite: (a) Fmoc-Trp-OH (the right figure shows the initial adsorption rate of Fmoc-Trp-OH), (b) Fmoc-Phe-OH, (c) Fmoc-Tyr-OH, (d) Fmoc-His-OH, and (e) Fmoc-Gly-OH. (f) Histogram showing the equilibrium loadings of Fmoc-Xs. (g) Histogram showing the initial adsorption rate of Fmoc-Xs.

Fmoc-X solutions studied except for Fmoc-Gly-OH which still had not reached equilibrium after 9 days (Figure 2).

The adsorption profiles contain two important characteristics of the adsorption process, i) initial adsorption rate, $k_{\mathrm{i}}$, which is a function of molecular diffusion rate to the fixed surface and sticking probability and ii) the final equilibrium concentration which is related to the surface coverage of the Fmoc-X and hence adsorption capacity of the surface for that species. As will be discussed later the Fmoc- $\mathrm{X}$ was in equilibrium between solution and adsorption; hence, these constants are expected to be a function of temperature, as at higher temperatures the increased $k_{\mathrm{B}} T$ will mean that more Fmoc-Xs will exceed their binding energy and enter solution.

It was found that Fmoc-Trp-OH had the highest affinity for graphite, which had an equilibrium loading of $0.12 \mathrm{mmol} / \mathrm{m}^{2}$ (Figure 2f). The other aromatic Fmoc-Xs had similar loading values, while the nonaromatic Fmoc-Gly-OH had a lower loading of $0.07 \mathrm{mmol} / \mathrm{m}^{2}$. The equilibrium loading was analyzed in terms of a range of parameters including the number of aromatic rings and $\mathrm{sp}^{2}$ carbons, solubility and hydrophobicity. 
However, no clear trend was found. The initial adsorption rate, $k_{i}$, was assumed to be constant for the very initial stages of the adsorption process. Therefore, $\mathrm{k}_{\mathrm{i}}$ could be expressed by the initial change in the amount of Fmoc-Xs on a unit surface area of graphite per unit time:

$$
k_{i}=\mathrm{d} n /(S \mathrm{~d} t)=-(V \mathrm{~d} C) /(S \mathrm{~d} t)
$$

where $n$ (nmol) is the number of Fmoc-Xs moles on graphite, $S$ $\left(\mathrm{cm}^{2}\right)$ is the surface area of graphite, $C(\mathrm{nmol} / \mathrm{L})$ is the concentration of Fmoc-Xs in solution, and $V(\mathrm{~L})$ is the volume of the solution. $\mathrm{d} C / \mathrm{d} t$ is obtained from the tangent to the adsorption profile at the initial linear phase. As seen in Figure $2 a$, the adsorption profile of Fmoc-Trp-OH showed linearity over the time range from 0 to 60 min with a coefficient of determination $R^{2}=0.99$. The initial adsorption rates for other Fmoc-Xs studied were derived using the same method (Figure $2 \mathrm{~g}$ ) and all the adsorption curves were found to display linearity over the same time range. Fmoc-Trp-OH and Fmoc-Tyr-OH had the highest initial adsorption rates with $k_{i}$ values of $\sim 0.23 \mathrm{nmol} / \mathrm{cm}^{2} \mathrm{~min}$ and Fmoc-Phe-OH showed the lowest rate $\left(k_{i}=0.01\right)$.

Adsorption of Fmoc-Xs on MWNT Mats. Our previous study demonstrated the successful dispersion of CNTs using Fmoc-terminated aromatic amino acids with the aid of ultrasonication. ${ }^{29}$ This work, though, concentrated on the dispersion of the nanotubes, rather than the adsorption of the Fmoc-X. Herein, immobilized MWNT mats which did not disperse in solution were used as a model surface so that the Fmoc-X remaining in solution could be easily followed. Figure 3 shows that the adsorption profiles of the Fmoc-Xs onto the MWNTs were similar to those onto graphite. A significant drop in the concentration of Fmoc-X in solution occurs within the first $100 \mathrm{~min}$, followed by a moderate decline until finally a constant value is obtained which is thought to be at equilibrium. Comparison of the data for the two surfaces showed that (i) the equilibrium loading of Fmoc-Xs on per unit area of graphite (Figure 2f) was much greater than that on the same area of MWNTs (Figure 3f) and that (ii) the initial adsorption rates of Fmoc-Xs on MWNTs (Figure 3g) were approximately 30 times slower than those on the graphite surfaces (Figure 2g). The relatively low equilibrium loading and initial adsorption rate on the MWNTs indicates the full surface area of the nanotubes was not accessible to the Fmoc-Xs possibly due to surface curvature effects or the pores between the nanotubes in the mats preventing diffusion of the amino acids to the lower portions of the nanotubes.

Freundlich Adsorption Isotherm of Fmoc-Xs on Graphite. The adsorption isotherms were studied for the Fmoc-Xs with the highest and lowest equilibrium loading on the graphite surfaces, Fmoc-Trp-OH and Fmoc-Gly-OH respectively (Figure 2f). We have shown previously through transmission electron microscopy that the Fmoc-Xs absorb heterogenously on graphitic surfaces such as carbon nanotubes. ${ }^{29}$ Furthermore, where the Fmoc-Xs do absorb, they tend to form multilayers $\sim 10 \mathrm{~nm}$ thick. These observations suggest that an adsorption isotherm such as the Langmuir isothermal is inappropriate for the Fmoc-X system as it describes the formation of a homogeneous monolayer, with the surface being saturated once that monolayer has been formed. The Freundlich isotherm equation was therefore used. This model based on the distribution of solute between the solid phase (the surface of an absorbent) and aqueous phase at equilibrium and can be derived through statistical mechanicals. ${ }^{35}$

(35) Yang, C. J. Colloid Interface Sci. 1998, 208, 379-387.
It is applicable to nonideal sorption on heterogeneous surfaces where multilayers can be formed and is given by

$$
Q=K C^{1 / n} \quad(n>1)
$$

where, for the current purpose, $Q\left(\mathrm{~mol} \mathrm{~kg}^{-1}\right)$ is the number of Fmoc-Xs adsorbed on unit weight of graphite and $C\left(\mathrm{~mol} / \mathrm{m}^{3}\right)$ is the final concentration of Fmoc-Xs in solution. $K$ is effectively the adsorption capacity (or adsorption power) of the surface for the surfactants and relates directly to how much the surfactants absorbs on the surface at equilibrium. $n$ is the adsorption intensity and is related to the lateral interaction between the adsorbed surfactant molecules and the heterogeneity of graphite surface which prevents this interaction. ${ }^{35,36}$ It should be noted that Freundlich isotherm does not predict saturation of the solid surface by the adsorbate, and therefore the surface coverage (i.e., $K$ ) is mathematically unlimited and includes the formation of multilayers. ${ }^{37}$

The equilibrium concentrations for Fmoc-Trp-OH and Fmoc-Gly-OH in the presence of graphite were measured for a range of initial concentrations between 0.02 to $0.08 \mathrm{mM}$. The adsorption capacity and intensity were calculated by linearizing eq 2 into logarithmic form as

$$
\ln Q=\ln K+1 / n \times \ln C
$$

and plotting $\ln Q$ against $\ln C$. Both Fmoc-Xs studied were found to follow the Freundlich isotherm model with a reasonable degree of fit (least-squares coefficient, $R^{2}>0.91$ ). Figure 4 shows these fit to the data and Table lgives the corresponding constants. The value of $K$ for Fmoc-Trp-OH $\left(1.04 \times 10^{-3} \mathrm{~mol}^{1 / 2} \mathrm{~m}^{2 / 3} / \mathrm{kg}\right)$ was 10 times that for Fmoc-Gly-OH $\left(9.02 \times 10^{-5} \mathrm{~mol}^{2 / 3} \mathrm{~m} / \mathrm{kg}\right)$, indicating the higher adsorptive capacity of graphite for Fmoc-Trp-OH than for Fmoc-Gly-OH. Molecular modeling has shown previously that the binding energy of the ionized Fmoc-Trp-OH on nanotubes is $47.7 \mathrm{kcal} \mathrm{mol}^{-1}$ whereas the binding energy of the ionized Fmoc- $\mathrm{Gly}-\mathrm{OH}$ is $36.2 \mathrm{kcal}$ $\mathrm{mol}^{-1}$. ${ }^{29}$ It was also found that the aromatic group in the Trp encouraged the Fmoc-Trp-OH to flatten against the nanotubes, following the tube's curvature, whereas the hydrophilic Fmoc$\mathrm{Gly}-\mathrm{OH}$ lifted away from the nanotube. Assuming that the general trends in relative binding energies and conformations are transferable to the planar graphite system studied here, it can be seen that the adsorption capacity increases with increasing binding energy. Another factor in $K$ in the Freundlich model is the ability to form multilayer films and it is plausible that the flattening of the Fmoc-Trp-OH aids multilayer formation.

For Freundlich isotherm, $n>1$ represents favorable adsorption conditions and thus both of the Fmoc-Xs were favorably adsorbed on graphite. n was greater for Fmoc-Gly-OH (3.06) than for Fmoc-Trp-OH (2.03) suggesting that the Fmoc$\mathrm{Gly}-\mathrm{OH}$ molecules interacted more between themselves within the absorbed layer.

Study of the Competitive Binding from a Fmoc-X Library onto Graphite. The previous experiments implied that the Fmoc-Xs were in dynamic equilibrium with the carbonaceous surfaces studied. Therefore, if a solution containing a library of different Fmoc-X species was used instead of a singular species, it would be expected that there would be competition to absorb on to the carbon's surface between the species. Over time it would be expected that the Fmoc-X with the strongest affinity for the

(36) Ng, C.; Losso, J. N.; Marshall, W. E.; Rao, R. M. Biores. Technol. 2002, 85, 131-135.

(37) Metwally, E.; El-Zakla, T.; Ayoub, R. R. J. Nucl. Radiochem. Sci. 2008, 9, $1-6$. 

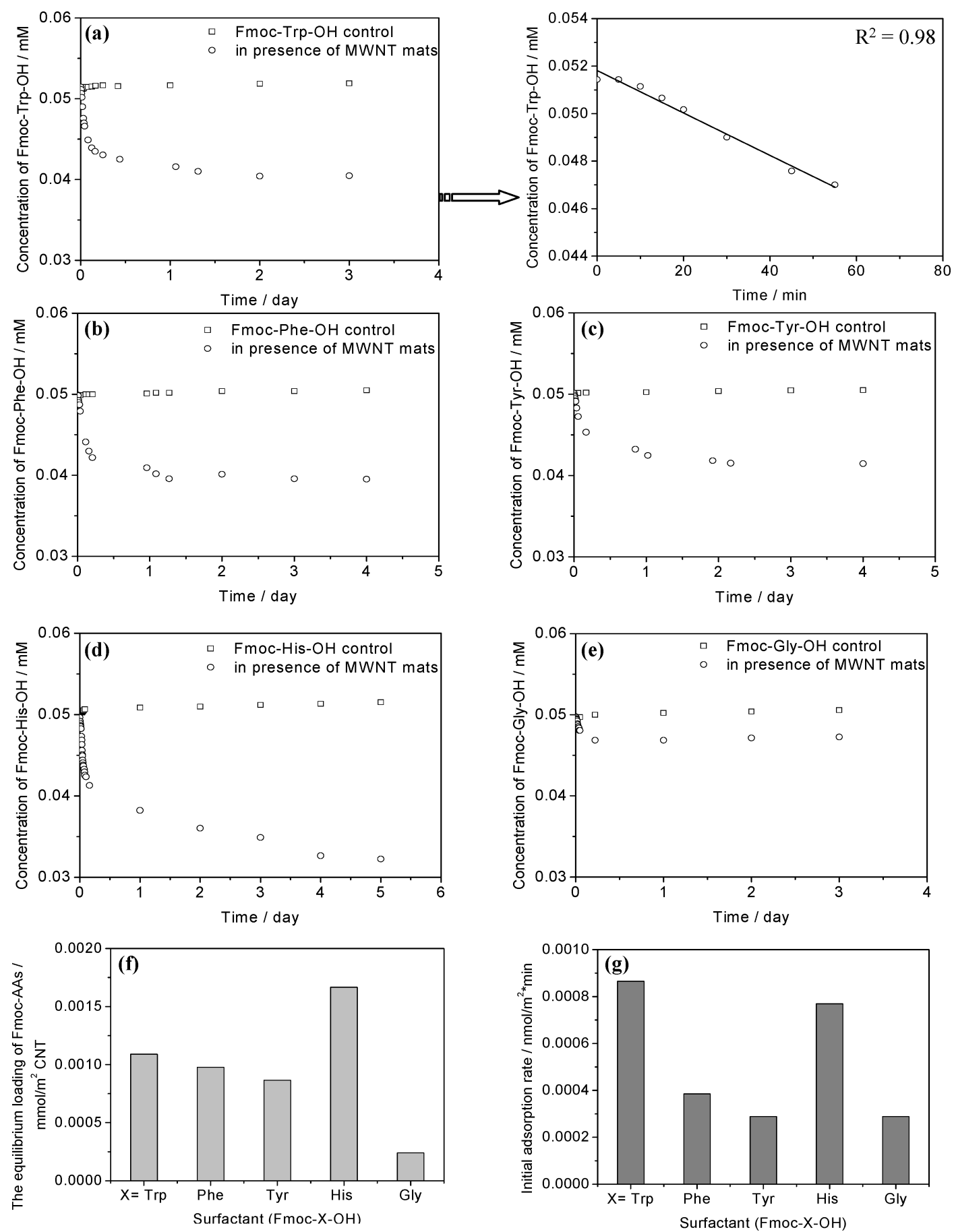

Figure 3. Adsorption profiles of the Fmoc-Xs on mats of aligned multiwalled carbon nanotubes: (a) Fmoc-Trp-OH (the right figure showed the determination of the initial adsorption rate of Fmoc-Trp-OH on the mat over the linear range of $0-60$ min), (b) Fmoc-Phe $-\mathrm{OH}$, (c) Fmoc-Tyr $-\mathrm{OH}$, (d) Fmoc-His-OH, and (e) Fmoc- Gly-OH. (f) Histogram showing the equilibrium loading of Fmoc-Xs. (g) Histogram showing the initial adsorption rate of Fmoc-Xs.

carbon surface would be predominately bound to the surface while those Fmoc-Xs with a poor affinity would be predominantly in solution. The advantage of developing such a competitive binding approach is that a number of Fmoc-Xs could be evaluated simultaneously in a very efficient manner.

The competitive experiments used a library consisting of five Fmoc $-\mathrm{Xs}(\mathrm{X}=\mathrm{Trp}-\mathrm{OH}$, Phe $-\mathrm{OH}, \mathrm{Tyr}-\mathrm{OH}$, His $-\mathrm{OH}$ and Gly- $\mathrm{OH}$ ) each at a concentration of $0.08 \mathrm{mM}$ and a target graphite substrate. (A higher concentration of Fmoc-Xs was used to ensure that there was a sufficiently strong signal for the analysis.) The concentration of the individual components in the library at a given time was analyzed using HPLC. The retention time for each Fmoc-X spacies was identified by using reference solutions of the individual species (Supporting Information). The library was then analyzed to give the baseline UV absorbance at $265 \mathrm{~nm}$ for each Fmoc-X component (Figure 5a). The library was introduced to the graphite surfaces and the concentration of each species was recorded as a function of time. The library was found to take $173 \mathrm{~h}$ to reach equilibrium and at this equilibrium the concentration of Fmoc-Trp-OH was significantly lower whereas the other Fmoc-Xs showed little change in their concentrations (Figure 5a). These data suggest that competitive 


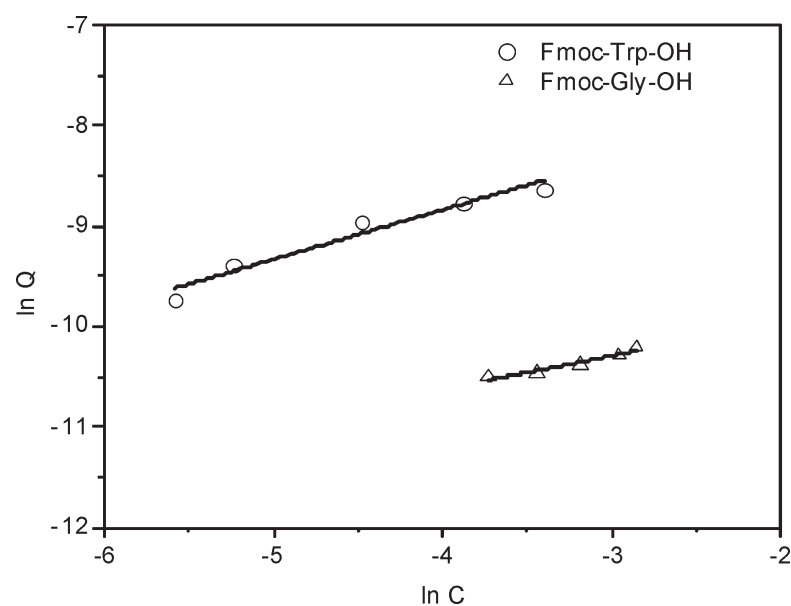

Figure 4. Plot of $\ln Q$ vs $\ln C$ for Fmoc-Trp-OH and Fmoc-Gly-OH absorbed onto graphite.

Table 1. Adsorption Capacity $(K)$ and Intensity $(n)$ from the Freundlich Isotherm Model for the Fmoc-Xs Adsorbed on Graphite ${ }^{a}$

\begin{tabular}{cccr} 
Fmoc-Xs & $K$ & $n$ & $R^{2}$ \\
\hline Fmoc-Trp-OH & $1.04 \times 10^{-3} \mathrm{~mol}^{1 / 2} \mathrm{~m}^{2 / 3} \mathrm{~kg}^{-1}$ & 2.03 & 0.93 \\
Fmoc-Gly-OH & $9.02 \times 10^{-5} \mathrm{~mol}^{2 / 3} \mathrm{~m} \mathrm{~kg}^{-1}$ & 3.06 & 0.91 \\
${ }^{a}$ Note that the units for $K$ depends on the value of $n$. The quality of fit,
\end{tabular}
$R^{2}$, is also given for each Fmoc-X. binding had taken place and Fmoc-Trp-OH was the dominant species adsorbed on graphite. The surface coverage of each component was calculated and compared to those found in the individual adsorption experiments (Figure 5b). It should be noted that the difference in the equilibrium surface coverage between the individual and library experiments was due to the higher initial concentration used in the latter. It can be seen that the individual experiments found that the aromatic Fmoc-Xs all absorbed at a similar level of $\sim 0.1 \mathrm{mM} / \mathrm{m}^{2}$, with no clear difference in their affinity for the graphite. However, in the competitive binding experiment, Fmoc-Trp-OH was clearly the strongest binder, with 3.5 times the coverage of the next strongest Fmoc-X (Fmoc-Phe-OH). The binding experiments also showed that libraries can be used to screen efficiently a wide range of binding candidates with similar binding energies. In these cases, the best binder is easily identified as its coverage is enhanced at the expense of weaker binders.

Sequential Displacement of Fmoc-Xs on HOPG Surface. Sequential adsorption experiments were conducted to verify that Fmoc-Xs with a higher binding energy could displace those with a lower binding energy on the surface. The displacement of Fmoc-Gly-OH (low binding energy) from a HOPG surface by Fmoc-Trp-OH (high binding energy) was followed using contact angle measurements. The contact angles on control HOPG surface and those immobilized with Fmoc-Trp-OH and Fmoc-Gly-OH were measured for reference. As shown in
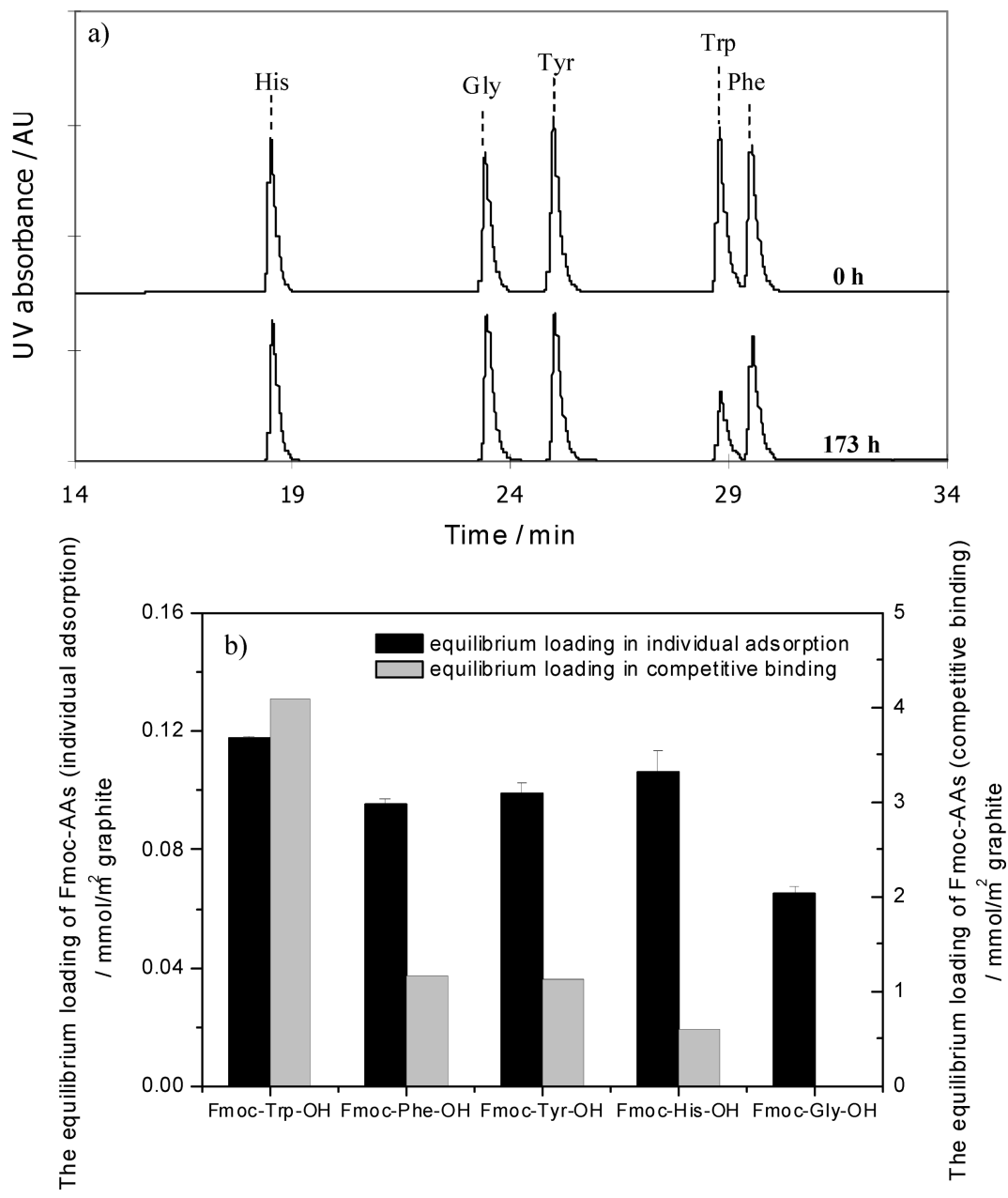

Figure 5. (a) HPLC traces of the mixture of the five Fmoc-Xs at $0 \mathrm{~h}$ (upper) and after $173 \mathrm{~h}$ of competitive binding (lower). (b) Comparison of the equilibrium loadings of the five Fmoc-Xs on graphite in individual adsorption and competitive binding. The data for the individual adsorption experiments is taken from Figure $2 \mathrm{f}$. 


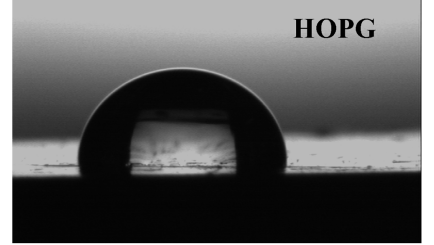

(a) $\theta=89.8^{\circ} \pm 2.8^{\circ}$

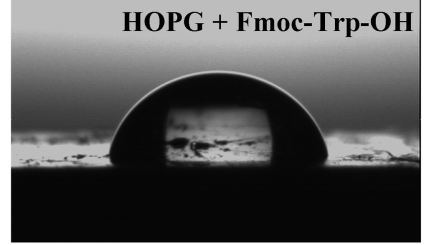

(b) $\theta=76.1^{\circ} \pm 0.7^{\circ}$

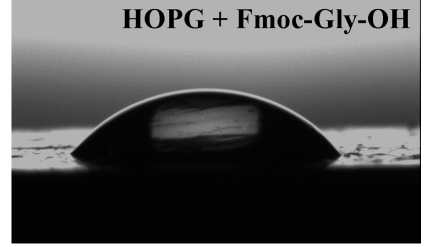

(c) $\theta=53.3^{\circ} \pm 1.6^{\circ}$

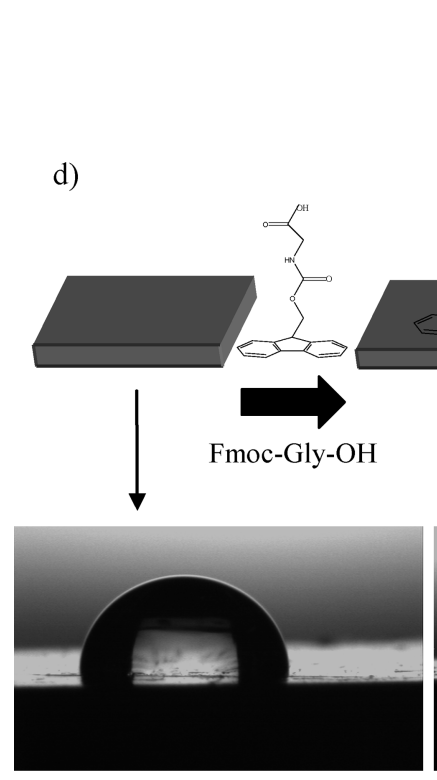

(e) $\theta=89.8^{\circ} \pm 2.8^{\circ}$

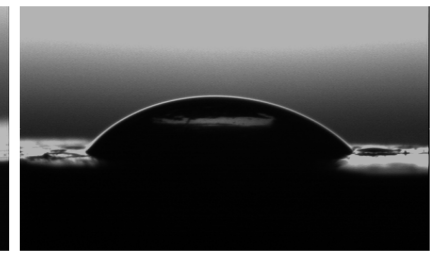

(f) $\theta=53.3^{\circ} \pm 1.6^{\circ}$

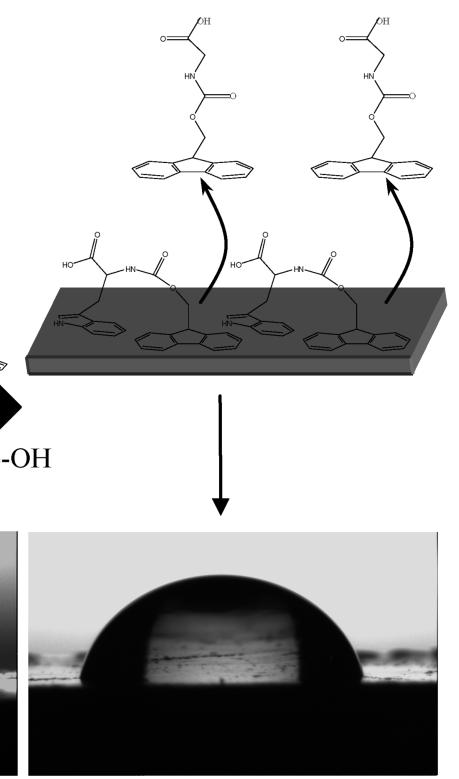

(g) $\theta=77.1^{\circ} \pm 1.1^{\circ}$

Figure 6. Contact angle measurements: (a) on freshly cleaved HOPG surface, (b) on Fmoc-Trp-OH functionalized HOPG surface, (c) on Fmoc-Gly-OH functionalized HOPG surface. (d) Schematic representation of the displacement of Fmoc- $\mathrm{Gly}-\mathrm{OH}$ by Fmoc-Trp-OH on the surface and corresponding contact angles for (e) HOPG, (f) Fmoc- Gly-OH functionalized HOPG surface, and (g) Fmoc-Gly-OH functionalized HOPG surface, which has been introduced to Fmoc-Trp-OH so that the Fmoc-Gly-OH has been partially displaced. Note that parts $\mathrm{c}$ and $\mathrm{f}$ are repeats of the same experimental condition.

Figure 6A, the contact angle on a freshly cleaved HOPG surface was $89.8^{\circ} \pm 2.8^{\circ}$ indicating its hydrophobic nature. After the adsorption of Fmoc-Trp-OH, the contact angle of the HOPG surface decreased to $76.1^{\circ} \pm 0.7^{\circ}$ due to the carboxylic group of the adsorbed Fmoc-Trp-OH. The contact angle for the surface functionalized with Fmoc-Gly-OH was lower $\left(53.3^{\circ} \pm 1.6^{\circ}\right)$ since Fmoc- $\mathrm{Gly}-\mathrm{OH}$ is more hydrophilic than Fmoc-Trp-OH.

The clear difference in contact angle between the two functionalized surfaces was then utilized to examine their sequential displacement on the surface. As can be seen in Figure 6B, after the HOPG that previously coated with Fmoc-Gly-OH was placed in Fmoc-Trp-OH solution for 2 days, the contact angle increased from $53.3^{\circ} \pm 1.6^{\circ}$ to $77.1^{\circ} \pm 1.1^{\circ}$, which was similar to that of the Fmoc-Trp-OH coated HOPG. This change in contact angle confirmed the successful displacement of FmocGly- $\mathrm{OH}$ by Fmoc-Trp-OH on the surface.

\section{Conclusions}

In summary, we have systematically investigated the dynamic adsorption behaviors of a series of Fmoc-amino acids on graphitic surfaces including graphite and MWNT mats. The adsorption equilibrium data were found to be described well by the Freundlich model. HPLC analysis helped to effectively screen for the optimal adsorbate in the competitive binding of these
Fmoc-Xs on graphite and the successful sequential displacement of Fmoc-Gly-OH by Fmoc-Trp-OH on HOPG surface was characterized using contact angle measurements. We believe that our study on the dynamic interaction between these aromatic amino acid derivatives and graphitic surfaces here provides a step forward for their application in biosensing where an effective and well-studied interface is required. There are also implications for the wider nanocarbon field, as it should be remembered that surfactants are in dynamic equilibrium. Hence, diluting the surfactant concentration or introducing a molecule with a stronger affinity can cause the surfactant to desorb, crashing the carbon dispersion. However, such an approach also gives the ability to recover the surfactant and clean the surfaces of the carbon after processing or sorting.

Acknowledgment. The authors thank the EPSRC, Royal Society, and Royal Academy of Engineering for funding. Y.L. also thanks Apurba Das for assistance with HPLC analysis, Zhe Li for providing MWNT mats and Mike Roberts for providing the HOPG sample.

Supporting Information Available: Figure showing HPLC spectra at $265 \mathrm{~nm}$ of the Fmoc-X library and the corresponding individual Fmoc-Xs. This material is available free of charge via the Internet at http://pubs.acs.org. 